Dass wir berechtigt sind, die Knochenfragmente auf den Foetus einer vor Jahren bestandenen Tubarschwangerschaft $z u$ beziehen, geht aus dem oben Gesagten einwandsfrei hervor. Um dieses zu erschliessen, ist die weitere Krankengeschichte gar nicht nöthig. Der einzige Einwand, dass dieselben mögiicher Weise einem macerirten Zwilling entstammen könnten, ist aus folgenden Gründen zurückzuweisen:

Die Grösse der Knochen zeigt, dass dieselben einem reifen oder wenigstens beinahe reifen Kinde entstammen, also von einem Foetus herrühren, der dieselbe Grösse besessen hat, wie der zweite vorgefundene, der keine Spur von Maceration zeigt.

Dass die Knochen von einem Foetus herstammen, der schon lange Zeit abgestorben und der Resorption ausgesetzt war, geht auch daraus hervor, dass von den Weichtheilen nur eine schmierige strukturlose Masse noch vorhanden war.

So weit mir bekannt geworden, dürfte vorliegender Fall sowohl in der pathologischen wie gynäkologischen Literatur ein Unicum sein. ${ }^{1}$ )

4.

\title{
Ueber eine enorme varicöse Geschwulst der linken Bauchwand.
}

\author{
Von Dr. med. A. Lejeune, \\ praktischem Arzt zu Frankfurt a. M.
}

(Hierzu Taf. IX.)

Zu Anfang dieses Jahres batte ich Gelegenheit, von einem Wreunde, Kreisphykus Dr. W. in S., zu einer Schiedsgerichtssitzung der Berufsgenossenschaft eingeladen zu werden. Unter den zu den Verhandlungen erschienenen Personen befand sich auch ein Mann, der wegen einer schlecht geheilten Fraktur anf Rente Anspruch erbob. Bei der körperlichen Untersuchung des betreffenden Mannes entdeckten wir einen grossen Tumor, der vow Nabel an über die linke Bauchseite hin sich bis zur Wirbelsäule erstreckte und ein enormes Convolut varcöser Venen darstellte. Da der Tumor von ganz ungewöhnlicher Grösse nnd Lage war, fasste ich schon damals den Entschluss, den Fall zu bescbreiben und erbat mir zu diesem $Z$ weck die Erlanbniss des Hausarztes jenes Mannes. Es sei mir an dieser Stelle gestattet, dem geebrten Herrn Kollegen Dr. T. in N. R. meinen aufrichtigsten Dank für die gütige Ueberlassung des Falles und seine mir noch ertheilten Auskünfte auszusprechen. Da ich bald daranf

1) Ausführlich im Arch. f. Gynäkologie publicirt. 
Vinchoro's Archivo, Bd, CLV.

Taf. IX.
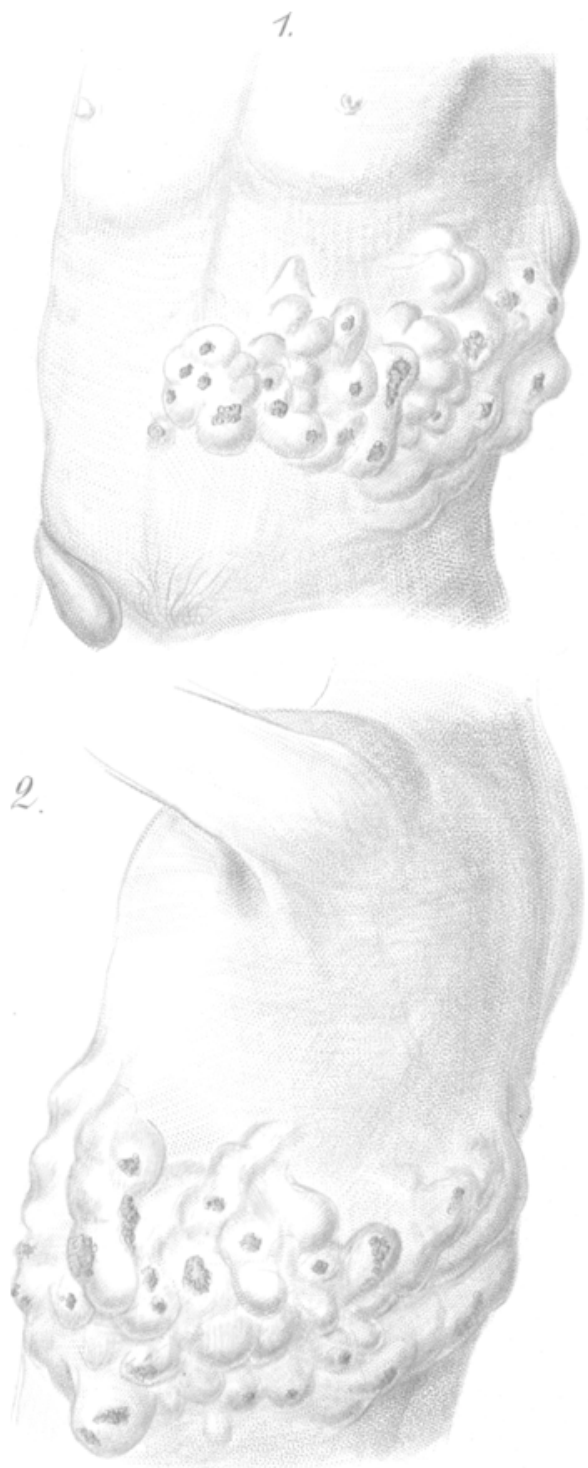

A. Lejoun det. 
die Gegend verliess, babe ich den Mann nicht mebr wiedergesehen; meine Beschreibung und die zwei Abbildungen beziehen sich auf. den Zustand, in dew ich den Kranken in Februar dieses Jahres zu Gesicht bekam.

Der Patient D., am 18. 8. 189857 Jahre alt gewesen, ist von Beruf Arbeiter und wohnt in N. R. Ueber die Art und Weise, wie der Tumor entstanden, kann er nichts genaues angeben; er meint, dass er angeboren sei und zwar als Naevus, der seiner Erinnerung nach ursprünglich die Grösse eines Markstückes gebabt baben soll.

Die Frage, ob in seiner Familie, sowobl aufsteigender, als auch absteigender Linie ähnliche oder gleiche Tumoren sich gezeigt baben, wurde negativ beantwortet, ebenso sollen auch nirgends in der Familie Naevi aufgetreten sein.

Während seiner Kindheit und auch später hat der Patient sich stets einer guten Gesundheit erfreut und von dem Tumor lange Zeit keine Beschwerden gebabt. Derselbe, bezw. der Naevus blieb stationär bis in die Mitte der Vierziger; da erst will Patient zum ersten Mal ein Wachsthum desselben bemerkt baben, es soll dasselbe aber sebr langsam von Statten gegangen sein. Als der Mann zum ersten Mal Beschwerden verspürte, war der Tumor schon von ziemlicher Ausdehnung in der Fläche, aber noch von unbedentender Böhe. Die Besebwerden waren hauptsächlich durch die Reibung der Kleidungsstücke auf der et«as empfindlicheren Haut über dem Tumor bedingt; eine Arbeitsbehinderung oder gar Unfähigkeit war aber dadurch nicht gegeben. Wie es kam, dass der Patient, trotzdem dass der Tumor sich langsam, aber stetig vergrösserte und erst geringe, dann erbeblichere Unannebmlichkeiten verursachte, sich nicht in cbirurgische Behandlung begab, war nicht zu eruiren; vielleicht hat er sich, wie das auf dem Lande leider so bäufig geschieht, von Kurpfuschern und sogenannten "weissen Frauen" vertrösten lassen, die Sache babe nichts auf sich, oder die Gesehwulst werde sich schon wieder zurückbilden, kurz, er hat nie den Versueb gemacht, so lange es noch Zeit war, den Tumor beseitigen zu lassen. Als Dr. T. und ich den Patienten zu Gesichte bekam, war es leider schon viel zu spät, als dass man mit Erfolg dem Fortschreiten hätte Einbalt thun oder gar den Tumor hätte beseitigen können.

Der Mann macht den Eindruck, als ob er früher sehr kräftig gewesen sei; der Knochenbau ist gut, der Thorax geräumig, jetzt durch chronisches Eophysem in der typiscben. Fassform, die unteren Rippen der linken Seite sind durch den wachsenden Tumor usurirt, besonders davon betroffen sind die beiden letzten Rippen. Wegen der Schmerzhaftigkeit der Palpation konnte die volle Ausdehnung der Usuren nicht festgestellt werden, doch unterliegt es wohl heinem $Z$ weifel, dass dieselben recht ausgedehnt und erheblich sind. Die Muskulatur ist ziemlich welk, ebenso soll der Panniculus adiposus eine Abnabme erlitten baben, doch ist dieser Schwund wohl bauptsächlich bedingt durch die vorhergehende lange Bettruhe wegen einer Fraktur, die der Mann vor einiger Zeit erlitten batte. Die Hautfarbe ist blass, Gesichtsausdruck normal, die Augen blicken etwas matt. Die sicht- 
barẹn Schleimbäute etwas blasser als normal, keine Cyanose. Puls und Temperatur normal.

Ueber den Lungen bört man vereinzeltes Rasseln und Schnurren, über den unteren Lungenpartien zumeist; die Spitzen sind beiderseits ganz frei von Geräuschen. Percussion und Auscultation des Herzens und der grossen Gefässe ergeben keine Anomalien, die Functionen des Herzens sind nicht beeinträchtigt. Sclerosen fehlen, Varicen an anderen Körperstellen sind nicht vorhanden, der Mann hat nie an Hămorrboiden oder Varicocoele gelitten.

Die Leberdämpfung ist in der Mamillarlinie etwas nach unten verlagert, in Folge des Emphysems und des Tiefstandes des Diaphragma, aber nicht verbreitert. Die Milzdämpfung ist durch Percussion nicht festzustellen, da der Tumor durch seine Lage eine solche unmöglich macht, doch ist eine Vergrösserung des Organs nicht sehr wahrscheinlich. Die Nieren und ibre Function zeigen ebenfalls keine pathologischen Veränderungen. Ueber den Tractus intestinalis werden keine Klagen laut, nur giebt der Patient an, beim Stuhlgang in der Geschwulst Schmerzen zu empfinden, die sich aber damit leicht erklären lassen, dass die Bauchpresse, die bei der Defäcation ja mitwirkt, z. T. durch die Contraction der Bauchmuskeln zu Stande kommt. Da nun in vorliegendem Falle die Bauchwand und die unteren Rippen durch den Tumor, wenigstens einseitig, ganz wesentlich verändert sind, tritt in Folge der Contraction der Bauebmuskeln eine bedeutende Staung des venösen Blutes auf; der Tumor fült sich prall mit Blut, und durch diese Ueberfüllung der Gefässe werden die Schmerzempfindungen ausgelöst, was auch dadurch bewiesen wird, dass der Mann die Spannung selbst beabachtet, über Schmerzen im After aber nie Klage geführt hat.

Rechterseits hat der Patient eine Hernia inguinalis, die durch ein gut sitzendes Bruchband zweckentsprechend zurūckgehalten wird.

Anamnestiscb ist noch von Wichtigkeit, dass der Mann weder bereditär, noch durch eigenes Verscbulden sypbilitisch ist; sowohl seine Angaben, als auch die objective Untersuchung lassen einen solchen. Verdacht als unbegründet erscheinen.

Nonmehr wende ich mich zu der Beschreibung des Tumor selbst. Icb erlaube mir, auf die beiden Abbildungen zu verweisen, die ich seinerzeit als Bleistiftsskizzen nach dem Leben anfertigte und nun, in Aquarell reproducirt, meiner Arbeit beifüge. Sie sollen einerseits als Erläuternng der Beschreibung dienen, andererseits dazu, den Fall nicht ganz der Erinnerung entschwinden zu lassen.

Der Tumor beginnt links vom Nabel, etwas oberbalb desselben und umzieht die ganze linke Bauchseite bis hinten zur Wirbelsäule. Nach oben hin, wie auch nach unten zeigt sich beine ganz scharfe Grenze gegen die nicht deformirten Tbeile des Abdomen; einzelne Varicen scblängeln sich über die Grenze des eigentlichen Convolutes hinaus und signalisiren schon im roraus, nach welchen Richtungen hin sich die Gefässveränderungen zu- 
näcbst wenden werden und wohin der Tumor wobl zunächst fortschreiten wird. So umzieht das Convolut der varicösen Venen die linke Brustseite in Form eines breiten Gürtels, dessen Länge $59 \mathrm{~cm}$ beträgt; gegenüber dem Umfang der anderen Seite, welcher blos $37 \mathrm{~cm}$ misst, eine Differenz von $22 \mathrm{~cm}$ zu Gunsten der erkrankten Bauchwand. Die Höhe des Tumors beträgt an der höchsten Erhebung, die sich in der vorderen Axillarlinie findet, $12 \mathrm{~cm}$. Die Breite des Varicengürtels wechselt zwischen $8 \mathrm{~cm}$ und $25 \mathrm{~cm}$, die breiteste Stelle findet sich ebenfalls in der genannten Linie, so dass wohl hier auch der Ausgangspunkt des ganzen Tumors zu sucben sein dürfte.

Die Farbe ist über dem grössten Theil des Tumors livide, mit einem Stich ins Bläuliche, an einigen Stellen ausserordentlich verdünnt; es haben auch bereits kleine Blutungen aus geplatzten Varicen stattgefunden, wenn auch nur unbedeutende. Wobl mehr als 50 kleine und grössere stahlblaue, höckerige, flache, cavernöse Angiome finden sich zerstrent auf dem grossen Tumor; meist sitzen sie den prominentesten Stellen als Varicen auf. "Keines von' ihnen ist gestielt oder yon anderer Farbe und Consistenz. Hinsichtlich der Grösse variiren sie zwischen Hanfkorn-, Linsen- und Bohnengrösse. Pulsation zeigt keines von ihnen, auf Druck entleeren sie sich ziemlich vollständig.

Die Druckempfindlichkeit des ganzen Tumors ist nicbt sebr beträchtlich. Es entsteht Athemnoth auf tiefen, auf einen grösseren Theil des Tumors lokalisirten Druck, ferner zeigt sich Stauung beim Niesen, Husten, Schreien u. s. w. An vielen Stellen des Tumors, besondere an den höchsten Stellen, ist Pulsation zu fühlen, ferner hört man systolische Töne und verschiedentliches Schwirren. Auch bereits durch die Grösse der Geschwulst besteht eine erhebliche Behinderung des Patienten bei allen Bewegungen. Eine Probe-Excision war nicht auszuführen, sowobl wegen der Gefahr einer eventuellen tödtlichen Blutung, als auch wegen der Decrepitität des Mannes.

\section{Nachtrag zu Dr. C. Davidsohn: Zur Erkennung zweier Stadien der Amyloiderkrankung.}

Während des Druckes der Arbeit starb eine zweite Maus, die in noch stärkerer Weise die amyloiden Veränderungen zeigte. Sie hatte in 7 Wochen 13 Injectionen erhalten, von 0,1 bis $1,0 \mathrm{ccm}$ steigende; Section und Untersuchung der Organe wurden sofort nach dem Tode ausgefübrt.

Bei makroskopischer Betrachtung war die Milz gross, (1,2 cm lang), glasig, hart; sonst nichts Auffälliges.

Mikroskopisch: in der Milz färbten sich die kernlosen, scholligen, breiten, ringförmigen Zonen in den Follikeln mit Jod roth, das übrige Gewebe gelb; nach Schwefelsäurezusatz traten genau, wie bei menschlichen Organen, alle Farbenübergänge vom-Violètt und Grän bis zum ausgesprochenen Veilchenblau auf, das Blau war so gesättigt, wie es eben 